\title{
Study on Optimum Usage of Power System upon Conventional Power Underwater Vehicle
}

\author{
Kun Yang ${ }^{\mathrm{a}, \star}$ and Lei Zhou ${ }^{\mathrm{b}}$ \\ College of Power Engineering, Naval University of Engineering, Wuhan, China \\ a 150006224@qq.com, ${ }^{b}$ 15345811275@163.coml
}

Keywords: Power system; Optimum usage; range; Endurance time

\begin{abstract}
For a conventional powered underwater vehicle, different use modes of power system have great influence on important technical performance indexes such as underwater range and underwater endurance time. First of all, this paper attempted to study the modeling method of the core energy module for the conventional power underwater vehicle power system, then based on the model of battery, the simulation model of underwater maximum endurance and maximum endurance time for underwater vehicle were established, and finally, some important conclusions about the general use of power system were obtained, which can provide scientific guidance for the performance evaluation, inspection and practical application of underwater vehicle power system.
\end{abstract}

\section{Introduction}

Usually in the everyday use of underwater vehicle, the commanders are often not concerned with the use of power system, but only consider the specific use of underwater vehicle tactics, in the same way, power system designers and users are often only concerned with the use of power system to meet the corresponding power requirements without considering tactical issues. This article attempts to combine the above two opinions, with the help of the theory and viewpoint of dynamics field, the optimum usage of the power system for the vehicle under the tactical background constraints is studied to guide the actual use of the power system.

Speed and endurance performance are the important technical index for underwater vehicle, in order to meet the requirements of different navigation targets, the commander of underwater vehicle is most concerned about how to estimate the actual endurance of the underwater vehicle at different speeds according to the current state of the specific power system[1-3]. Therefore, it is necessary to carry out the study on the optimum usage strategy of the power system, so as to make the underwater vehicle get better economic and endurance performance under the same conditions. Due to the kind of underwater power supply module for traditional diesel electric power underwater vehicle only has battery, therefore, the modeling of the battery module for the vehicle becomes one of the most important tasks in the power system study.

\section{Model Establishment of Battery Energy Module for Underwater Vehicle}

As the energy storage system of a conventional underwater vehicle, the battery is one of the most important problems to be solved in the modeling of underwater vehicle power system. When the battery is sailed by underwater power supply, as the discharge continues, the voltage of battery keeps dropping while the current of battery keeps rising, and there is almost no law to follow, so it is very difficult to simulate the voltage and current change with time directly. For model establishment of battery which is for the purpose of application, it not only requires that it can better reflect the discharge dynamic process of the battery, but also requires that it can not be too complex to convenient for engineering applications. In view of the complexity of the electrochemical mechanism of battery, starting from the study of the external characteristics of battery, the improved neural network algorithm combined with one-dimensional search algorithm is proposed to solve the problem of battery model establishment in this paper. 
Analysis of Actual Discharge Process Characteristics for Battery. Under normal circumstances, conventional underwater vehicle maintain a constant speed under water, that's to say, the power to push the hull maintain a constant, at the same time, the power of air conditioning and other auxiliary machineries also maintain a constant value, Therefore, the discharge process of the battery is considered as a quasi-static constant power discharge process with constant power value in this paper. And through the analysis, we can see that the actual battery constant power discharge curve is steeper than the constant current discharge curve, so at any moment the discharge process, the constant power discharge curve must intersect with a constant current discharge curve, and if the voltage and current value of the constant current discharge curve can be obtained, the voltage and current value at the corresponding time of actual constant power discharge curve of the battery also can be obtained, in the same way, the constant power discharge model of the storage battery can be obtained by searching the current and voltage values at different discharge points on the constant current discharge curve.

The Model Establishment of Battery Constant Current Discharge Process. Limited by the life and cost of battery for underwater vehicle, a lot of tests can not be carried out to provide sufficient data for modeling, and only a limited number of discharge test curves can be used to model them, therefore, in order to search a series of equal power points from the infinite constant current discharge curve, it is necessary to consider extending the limited test data curves to any number of curves, The model of constant current discharge under the condition of arbitrary discharge rate at different discharge cycles is established, that's to say, it is necessary to establish the model of constant current discharge under the condition of arbitrary discharge rate at different discharge cycles, and in this paper, a constant current discharge model of battery under arbitrary discharge rate is established by neural network.

Based on constant current discharge test curve data, an improved Levenberg-Marquardt algorithm[4-9] is used to write programs for solving the law of constant discharge under arbitrary discharge rate, the design network structure is shown in Fig. 1, the network contains three layers: the input layer, the hidden layer, and the output layer, the hidden layer transfer function selects the tansig function, the output layer selects the purelin function, the network input vector is $X=\left(x_{1}, x_{2}, x_{3}, x_{4}\right)$ ( Discharge period, discharge rate, discharge time, initial voltage), the network output is the battery terminal voltage at different discharge time of different discharge rates during a discharge period, so the number of network neurons in the input and output layer is 4 and 1 respectively.

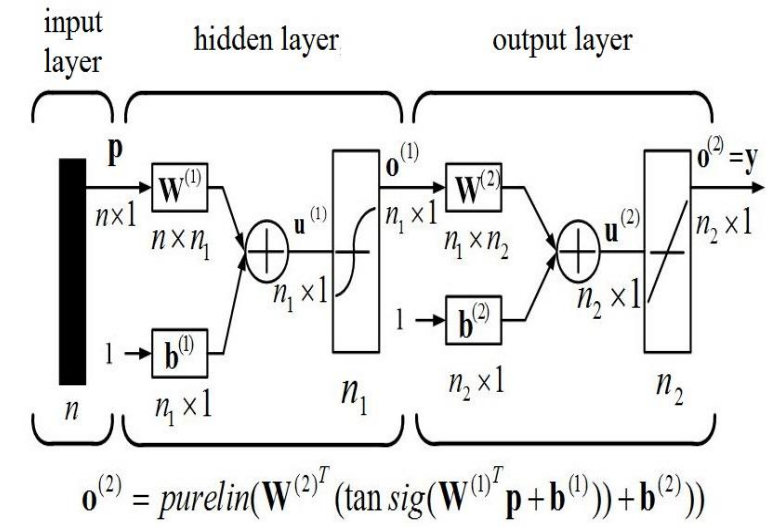

Figure 1. Structure of the network

In addition, in order to make the network have good generalization ability[10-13], the cross validation method is used to prevent the phenomenon of Network over training in this paper, the specific procedures flow chart is shown in Fig. 2. To facilitate monitoring of the entire network training process, the program plots the error variation curve of the subsets for the network learning samples which is shown in Fig.3, as we can see from the Fig. 3, At the beginning of training, the errors of the three subsets both decrease, however, when the network has been over trained, the error of confirmation subsets starts to rise, and when the ascending continuous number of confirmation error 
exceed the limit, the training terminates, finally the network weight parameter corresponding to the minimum location of the confirmation error returns. In addition, it also can be seen from Fig. 3 that the confirmation subset error curve is basically consistent with the test subset error curve, which shows that the division of the data sample set is reasonable.

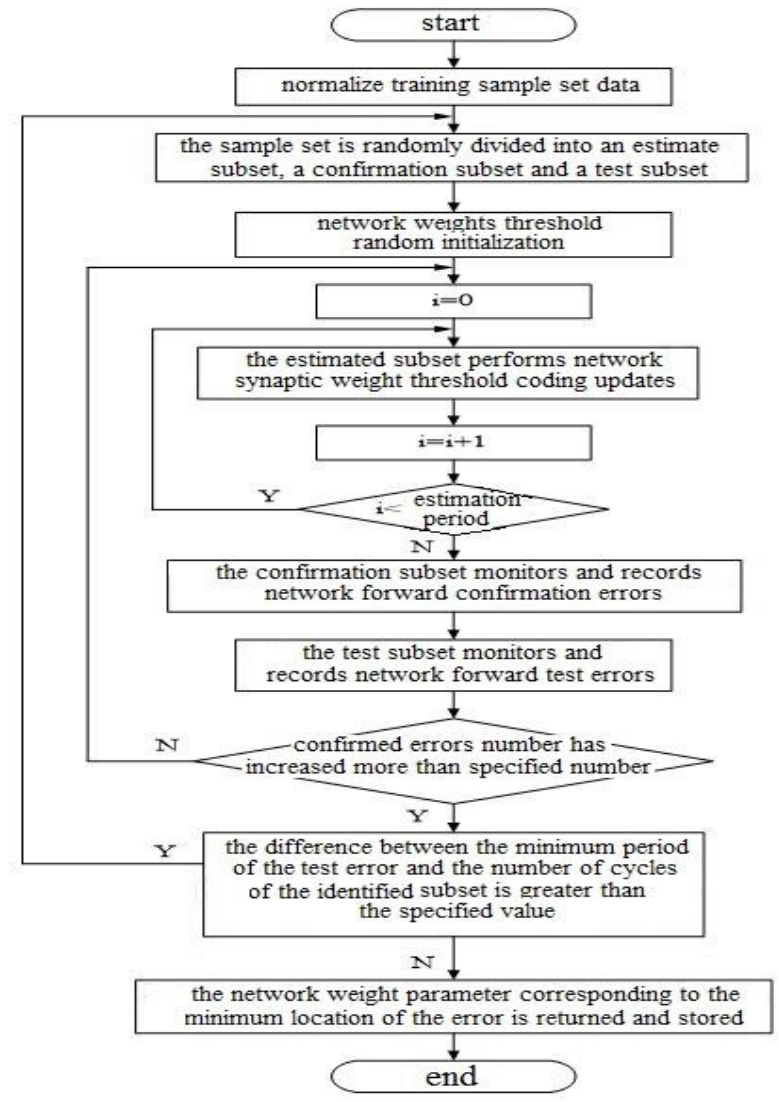

Figure 2. Network cross-validation program flow chart

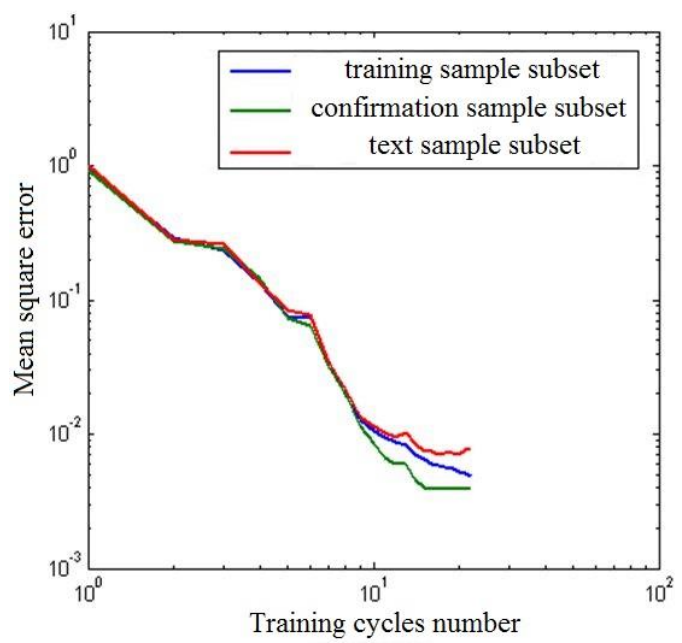

Figure 3. Network cross-validation error variation curve

After further analysis of the network response, the output of the network is normalized, and the network actual output as well as the corresponding target response are analyzed by linear regression, it can be seen from Fig. 4 that the network output tracks the target response well, which demonstrates that model establish is successful. The updating process of network partial weights is shown in Fig. 5. 


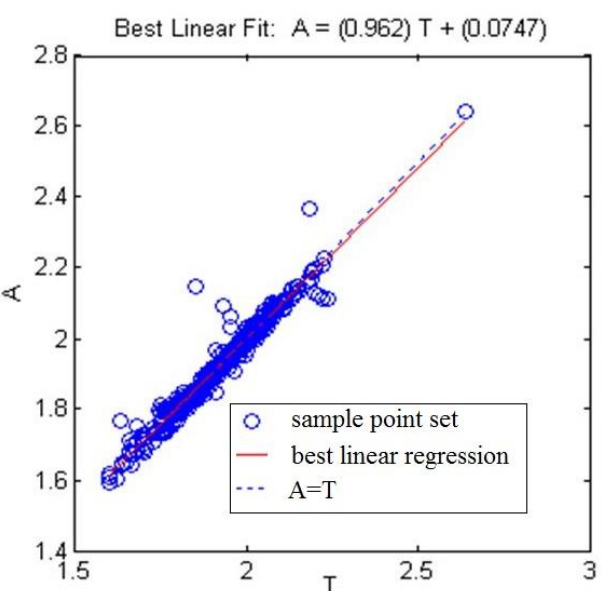

Figure 4. The Linear regression analysis of network output and response

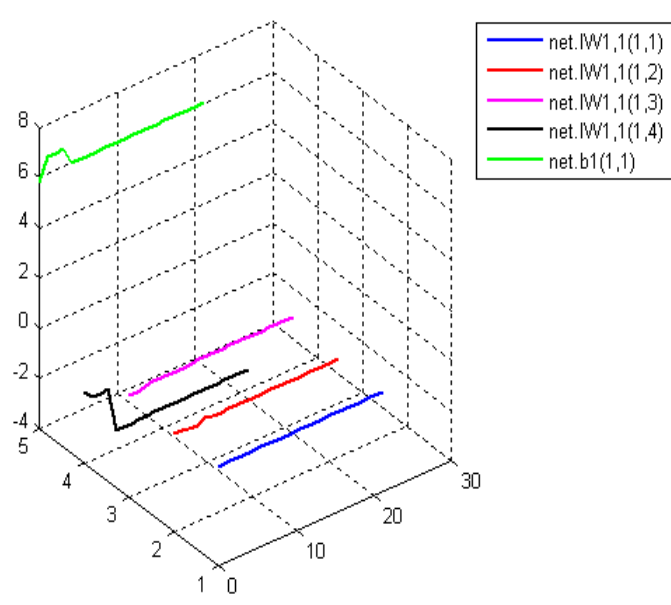

Figure 5. The change of some weights of the network in the search space

Considering that the actual constant power discharge curve decreases monotonically, therefore, there is only one constant current discharge curve that intersects the constant power discharge curve at any intermediate moment, searching the point corresponding to the given power value in numerous points on the constant current discharge curve at any intermediate moment according to the power balance relationship, and this point is the intersection of the constant power discharge curve and the constant current discharge curve at this time. Based on the constant current discharge model, and the constant power discharge model of the battery can be obtained via searching a series of equal power points in the constant current discharge curve by using one-dimensional search algorithm. The constant power discharge curve corresponding to the discharge power of $200 \mathrm{~kW}$ is shown in Fig. 6, and the constant power discharge curve with arbitrary power value can be obtained by varying power values in a program dynamically.
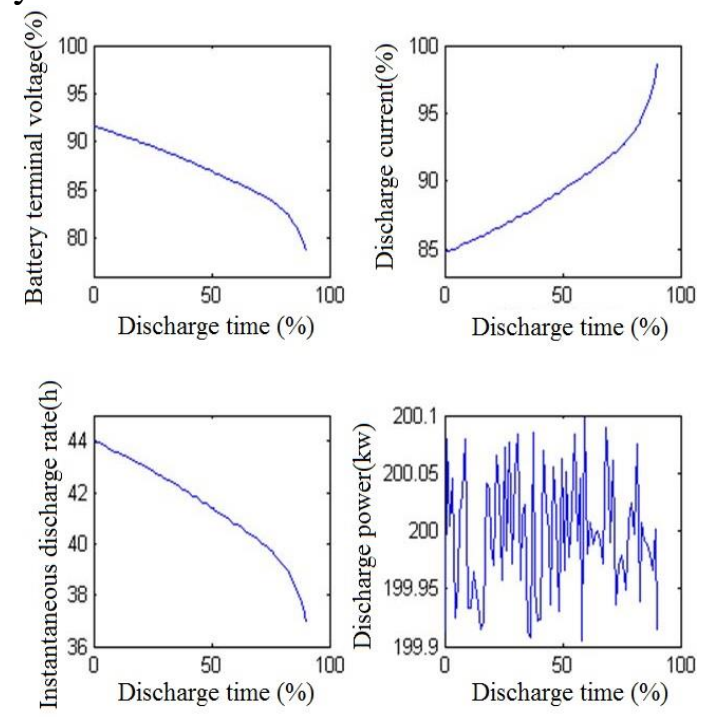

Figure 6. Network cross-validation error variation curve

\section{The Model Establishment and solution of Range for Single Navigation}

Underwater submerge maximum range of underwater vehicle refers to the maximum distance that can be reached in an underwater discharged and charged navigation when the vehicle battery does not carry out the charging of the snorkel, It is one of the important indexes to measure the tactical performance of vehicle. The single submerge maximum range model is used to break through the enemy long distance and reconnaissance area for underwater vehicle, The optimization result can 
make the underwater vehicle have a longer underwater single submerge range and endurance time, so the underwater vehicle can pass through the narrow and dangerous area of the enemy smoothly.

$S_{B} \max =v_{B} \times t_{B}=v_{B} \times \Lambda\left(v_{B}, \eta_{a}, p_{a}\right)$

Among them:

$S_{B}$ : Underwater submerge single range of underwater vehicle

$\eta_{a}:$ Transmission efficiency of shafting

$p_{a}:$ Auxiliary machineries power consumption

$v_{B}:$ Battery propulsion speed

$t_{B}:$ Battery propulsion navigation time

$\Lambda:$ The relation between the parameters described in the battery propulsion and the navigation time of underwater vehicle, which is mainly determined by the model of the battery discharge process.

The underwater power supply propulsion speed restraint of battery:

$a 1 \leq v_{B} \leq a 2$

Among them:

$a 1$ : The minimum navigation speed for maintain the stability of underwater navigation for underwater vehicle accumulator

$a 2$ : The maximum navigation speed of underwater vehicle accumulator for underwater navigation

When an underwater vehicle navigates at sea, the long time complete charging level can not be achieved because of the hidden need(Generally can only achieve normal charging level), Therefore, the electrolyte density at the discharge starting point is generally no higher than the battery electrolyte density at the normal charging ending point. In addition, to ensure that the underwater vehicle can cope with unexpected situations at any time, the battery should keep a certain proportion of the remaining electricity at any time, therefore, the remaining electrolyte density of the battery should be higher than a certain proportion of remaining value. The case of the remaining electricity amount with $30 \%$ is considered in this paper.

When solving the Eq. 1 by combining the battery constant power discharge model and jet propulsion model, the following results can be obtained:

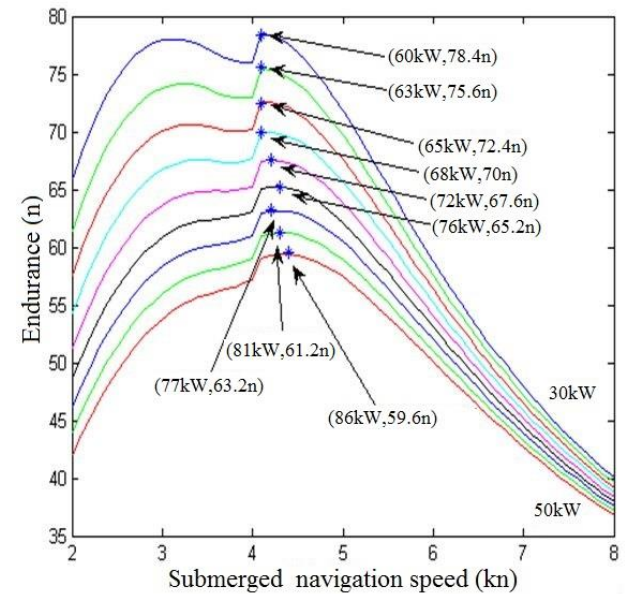

Figure 7. The range curve family at different navigation speed

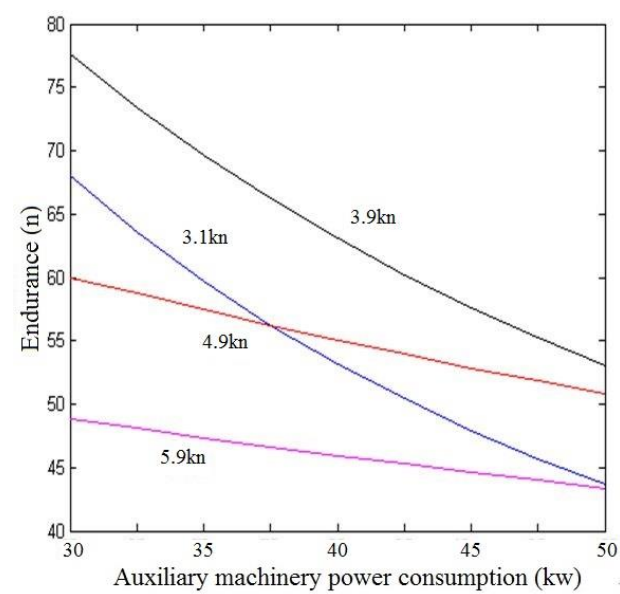

Figure 8 . The range curve family at different auxiliary machinery power

It can be seen from the Fig. 7 and Fig. 8 that it has global maximum points in the whole course of navigation speed, and these operating points are all located near the navigation speed of the underwater vehicle, it also has local maximum point in the whole course of navigation speed, and the endurance index decreases with the increase of the auxiliary machineries power. It can be seen from the Fig. 9 and Fig. 10 that the endurance time of underwater vehicle tends to decrease with the increase of the auxiliary machinery power and speed. 


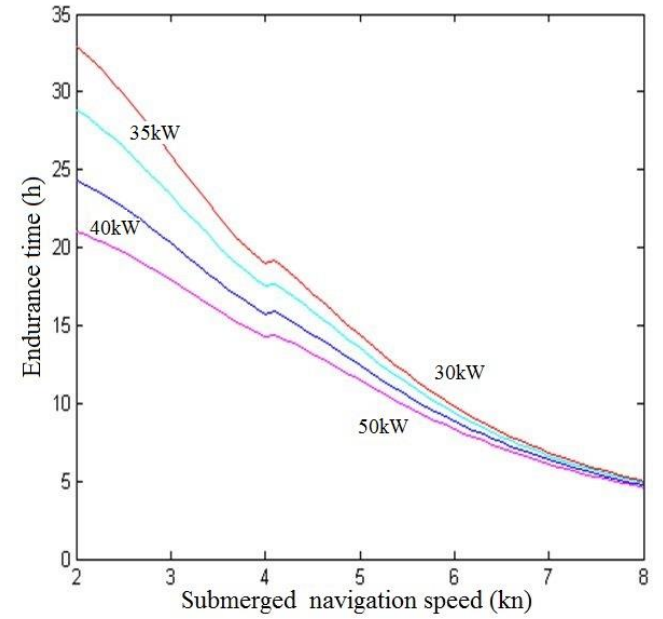

Figure 9. The endurance time curve family at different navigation speed

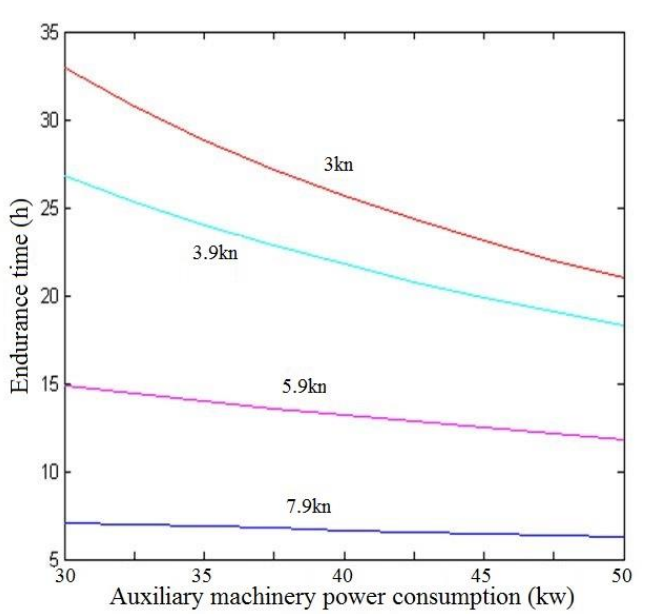

Figure 10. The endurance time curve family at different auxiliary machinery power

The range and endurance time nephogram under different auxiliary machinery power and navigation speed are shown in Fig. 11 and Fig. 12.

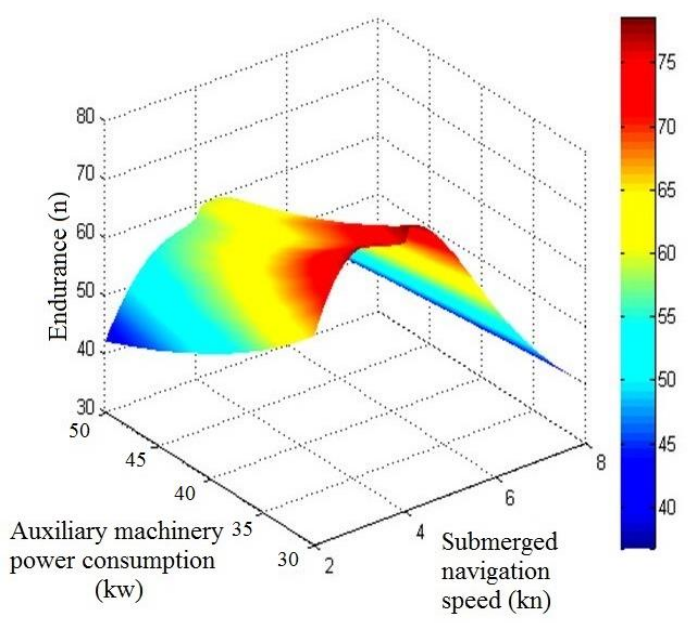

Figure 11. The range nephogram under different auxiliary machinery power and navigation speed

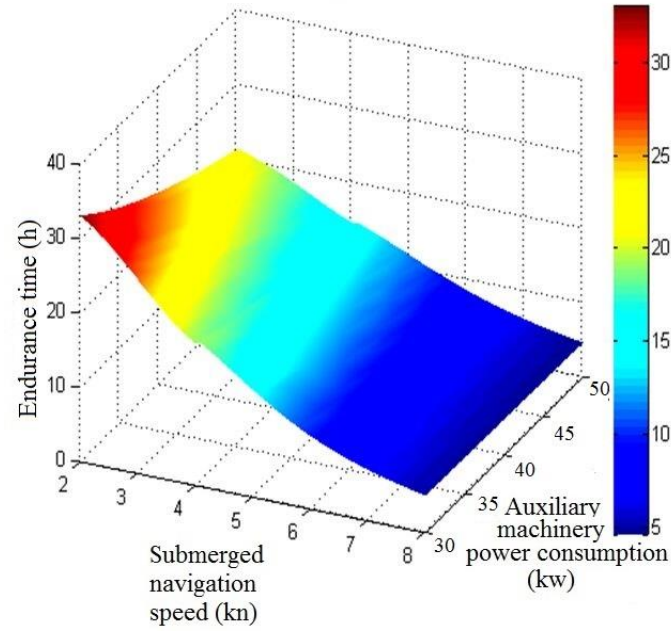

Figure 12. The endurance time nephogram under different auxiliary machinery power and navigation speed

The range curve family of underwater vehicle when encounter with ocean current under $75 \mathrm{~kW}$ and 60kW auxiliary machinery power are shown in Fig. 13 and Fig. 14, it can be seen from the Fig.14 and Fig.15 that the influence of ocean current and auxiliary machinery power on the underwater vehicle endurance is very obvious, and when the ocean current is downstream, the navigation speed should reduce appropriately, when the ocean current is upstream, the navigation speed should increase accordingly to obtain the maximum endurance index. 


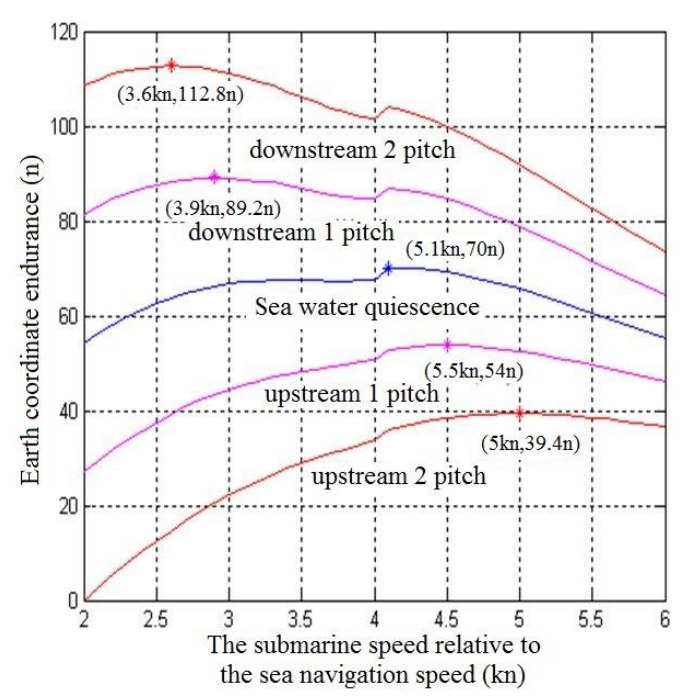

Figure 13. The range curve family of underwater vehicle under $37 \mathrm{~kW}$ auxiliary machinery power

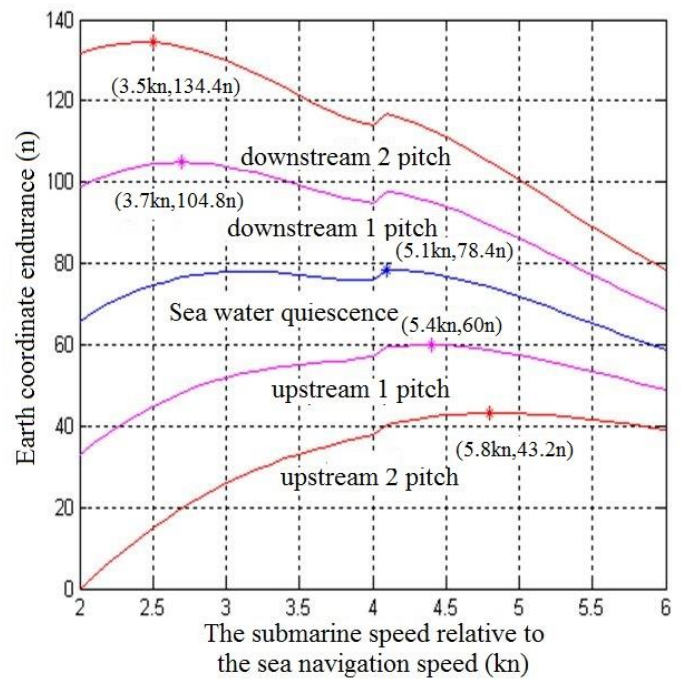

Figure 14. The range curve family of underwater vehicle under $30 \mathrm{~kW}$ auxiliary machinery power

\section{Introduction}

Based on the detailed analysis to external characteristic data of underwater vehicle battery discharge process, the method which uses improved neural network algorithm combined with one-dimensional search algorithm to establish the battery discharge process model is proposed in this paper. And this method can solve the difficult problems in the process of establishing the battery model successfully, which also has been used in practice.

The underwater vehicle has global maximum points in the whole course of navigation speed, and these operating points are all located near the economic navigation speed of the underwater vehicle

When the underwater vehicle encounters with ocean current, the vehicle speed relative to the sea navigation speed should reduce appropriately when the ocean current is downstream, and the vehicle speed relative to the sea navigation speed should increase when the ocean current is upstream, at the same time, the impact of auxiliary machineries power consumption should be reduced to maximize the range.

Due to the influence of auxiliary machineries power consumption on range at various conditions is very large, it is necessary to carry out study on the auxiliary machinery energy saving, such as adopting an auxiliary machinery with higher energy conversion efficiency, using high voltage system to reduce network loss and closing some unnecessary electrical appliances on the navigation to save electricity, et al. In short, try to reduce the auxiliary machinery power consumption for vehicle can improve the range effectively, it also can reduce exposure rate effectively at the same navigation speed, and the effect is particularly obvious for vehicle at low speed and micro speed navigation.

\section{Acknowledgements}

The work is supported by National Natural Science Foundation of China (51409254).We are grateful for their financial supports.

\section{References}

[1] F.Q.Liu, D.M.Xu, Gao Jian, et al. Fault Diagnosis and Fault Tolerant Control for Actuators of Underwater Vehicles [J]. Control Theory \& Applications,2014, 31 (9):1143-1151.

[2] D.Q.Zhu, Z.Hu. Fault Diagnosis and Fault Tolerant Control for the Underwater Robot [M]. Beijing: National Defense Industry Press, 2012. 
[3] J.Gao, F.Q.Liu, J.Zhao, et al. Non-linear Path Following Control of Underactuated Autonomous Surface Vehicles [J]. Robot, 2012, 34(3): 329 - 336.

[4] N.Samani, M.Gohari and A.A.Safavi. A Simple Neural Network Model for the Determination of Aquifer Parameters[J]. Journal of Hydrology, 2007, 340(1-2): 1-11.

[5] Y.F. Gao, Y.Sun and W.J. Zheng. Genetic Algorithm for Optimizing the Layout of Logistics Park Based on Shortest Path and Elitist Strategy[J]. Journal of Information and Computational Science, 2015,12( 10) : 3765- 3774.

[6] C.H. Dong. Neural network and Applications[M]. Beijing: National Defense Industry Press, 2005.

[7] H.R. Liu, C.X. Zhao, X.Li, et al. Troy Nagle H. Study on a Neural Network Optimization Algorithm Based on Improved Genetic Algorithm [J]. Chinese Journal of Scientific Instrument, 2016, 37(7): 1573-1580.

[8] M.Jalali, M.Asadollahi, P.Shahbazikhah. QSAR Study of Heparanase Inhibitors ActivityUsing Artificial Neural Networks and Levenberg-Marquardt Algorithm[J]. European Journal of Medicinal Chemistry, 2008, 43(3): 548-556.

[9] S.Vijander, G.Indra and H.O.Gupta. ANN-based Estimator for Distillation Using Levenberg-Marquardt Approach[J]. Engineering Applications of Artificial Intelligence, 2007, 20(2): 249-259.

[10] I.Sébastien, P.G.Jean. Computer Aided Optimal Designs for Improving Neural Network Generalization[J]. Neural Networks, 2008, 21(7): 945-950.

[11] Y.Y. Yu,Y.Chen,T.Y.Li, et al. Improved Genetic Algorithm for Solving TSP[J]. Control and Decision, 2014, 29( 8) : 1483- 1488.

[12] W.S.Wan, M.Shingo and S.Kaoru. Enhancing the Generalization Ability of Neural Networks Through Controlling the Hidden Layers[J]. Applied Soft Computing, 2009, 9(1): 404-414.

[13] B.J. Cai. Speed Observe of Direct Torque Control Based on GA and BP Network [J].Control Engineering of China, 2012, 19(4) : 733- 736. 